\title{
Das interkulturelle Potenzial von Karikaturen im DaF-Unterricht
}

\author{
The intercultural potential of caricatures \\ in classes of German as a foreign language
}

\begin{abstract}
Pictures/caricatures - as a part of culture are characteristic for a society (nation). In the process where different cultures get in touch pictures may play a role in teaching a foreign language. Introduction into an intercultural topic, intensive discussion - there are many possibilities for a teacher to show his/her students the life in a German-speaking country in its various facets. Caricatures can combine politics, society, religion, culture and sense of humour with an enrichment of the language used in classroom - at the same time supporting the development of the intercultural competence of the students.
\end{abstract}

KEYWORDS: caricature, interculturality, classroom, intercultural competence, culture, Germanspeaking country.

\section{INTERKULTURELLE KOMPETENZ - INTERKULTURELLES LERNEN}

Viele Lehrer, Lektoren und Vortragende machen sich Gedanken darüber, wie und in welcher Form die interkulturelle Kompetenz der Lernenden durch einen entsprechenden Fremdsprachenunterricht gefördert und gestärkt werden kann (über eventuelle andere als die hier vorgestellten Möglichkeiten vgl. Utri 2008, 2009a, 2009b, 2010a, 2010b), denn, wie F. Grucza (1993) schon früh bemerkte, sollte diese Art der meist auf Sprache fundierten Kompetenz einen unverzichtbaren Teil des Fremdsprachenunterrichts ausmachen. 
Die Sprachdidaktik spielt sich nicht im „Elfenbeinturm” ab, sie soll auch nicht abgehoben von der Realität, das heißt von der jeweiligen Kultur, sein, sondern kann auch national geprägt sein, auch durch die jeweilige Sprache - genauer gesagt Texte aus einem der deutschsprachigen Länder (vgl. Utri 2007: 226). Gerade mit sprachlichem Material, das heutzutage in Form authentischer Texte bzw. Bilder in großen Mengen zur Verfügung steht, kann man die interkulturellen Fähigkeiten/Kompetenzen entwickeln helfen. Auch auf der Metaebene sollte diese Problematik genauer behandelt werden - die Wissenschaft, egal ob theoretisch oder praktisch didaktikorientiert - habe die Aufgabe, den interkulturellen Kompetenzen nachzugehen, um

Probleme der Menschen und der Gesellschaft zum Thema von Wissenschaften $\mathrm{zu}$ machen, wie auch Ergebnisse von Wissenschaft in den gesellschaftlichen Diskurs einzubinden (H.-J. Krumm 2003: 9).

Die jeweilige Sprache und die jeweilige Kultur des Landes prägen oft auch das wissenschaftliche Denken und Argumentieren und damit indirekt auch die jeweilige Didaktik.

Allerdings sollte man sich angesichts des in der DaF- bzw. DaZ-Literatur der letzten Jahre auftretenden Modewortes „interkulturell” eingehender damit beschäftigen, was dieser Begriff eigentlich bedeutet. Das Adjektiv interkulturell kommt schon haufenweise in unterschiedlichen Kontexten vor, mittlerweile wird einerseits postuliert, dass es bei der interkulturellen Kommunikation (und deren Vorbereitung, also dem interkulturellen Lehren und Lernen) noch viele ungelöste Probleme gebe (vgl. Röttger 2010: 7), andererseits wird schon von einem „Kollaps des Interkulturalitätsparadigmas" (Altmayer in Röttger, ibidem) gesprochen. Röttker kritisiert zurecht die Tatsache, dass es im deutschsprachigen Sprachraum nur wenige Ansätze für die Entwicklung von interkulturellen Lehrwerken gegeben hatte und dass der Begriff „Interkulturalität” im angelsächsischen Sprachraum von Anbeginn mit "Landeskunde” (engl. cultural studies) verwechselt worden war.

Die Tatsache, dass nicht alles, was „kulturell” ist, auch interkulturell bedeutet, sollte vor allem im theoretischen Diskurs über die Interkulturalität und das Interkulturelle Lernen unbedingt berücksichtigt werden. Die meisten Sprachlehrer sind sich darüber einig, dass die Reflexionsfähigkeit sowohl der Lehrenden als auch der Lernenden notwendige Grundvoraussetzung sei und heutzutage für ein effektiveres Lernen unverzichtbar sei. Im allgemeinen Lernbereich spricht man vom Bewusstsein des eigenen Lernens, im sprachlichen Bereich spricht man von "language awareness" 
(Sprachbewusstheit), was ja sowohl die Muttersprache(n) als auch die Zweit- oder Drittsprachen einschließt.

So wie ein bilingualer Mensch von einer Sprache in die andere "switchen" kann, so ist eine Person, die mit entsprechender interkultureller Kompetenz ausgestattet ist, in der Lage, von einem Kulturbereich zum anderen (in dem dann andere Normen, Traditionen, Werte und Gebräuche Gültigkeit haben) zu switchen (vgl. Grucza 2006: 42; an dieser Stelle erklärt er auch, warum die interkulturellen Eigenschaften eines Menschen die multikulturellen einschließen). Es herrscht Einigkeit darüber, dass im Rahmen des Zweitsprachenerwerbs die Entwicklung einer interkulturellen Kompetenz von entscheidender Bedeutung sei. So meint Pfeiffer (2001):

Die rein sprachliche Kommunikation wird durch die interkulturelle Kommunikation, die Landeskunde durch integrierte, interkulturelle oder erlebte Landeskunde ersetzt oder zumindest ergänzt. Als Lehrziel und Lehrprinzip gilt nun die interkulturelle Spracherziehung.

Interkulturelle Kompetenz beinhaltet das Wissen um eine andere Kultur (und die damit verbundenen Sinnzusammenhänge der jeweiligen Kulturgemeinschaft) sowie auch die Anwendung im praktischen Sinne. Dies muss jedoch nicht unbedingt auf ein Land beschränkt sein. So kann es einerseits ähnliche/gleiche Traditionen in z.B. Deutschland und Österreich geben, andererseits kann es in einem Land sehr unterschiedliche Traditionen (die auch mit unterschiedlichen Sprachen verbunden sein können) geben. So wäre das gegenseitige „Begutachten”, (Kennen)Lernen und eventuell auch Wertschätzen von Sprache (in Form von Texten) bzw. anderen kulturellen Äußerungen/Erscheinungen (darunter verstehe ich sowohl erfahrbare Handlungsschemata - Rituale, Sitten und Gebräuche, übliche/normierte Handlungsmuster bzw. -abfolgen, welche Ausdruck von Werten und z.T. nicht bewusst gemachten, internalisierten Kommunikationsmustern darstellen, als auch jegliche Artefakte/kulturelle Erzeugnisse, die landläufig als Bestandteile der Kultur oder als die schönen Künste gelten) zwischen Schlesiern, Kaschuben und sonstigen Polen auch eine Form des interkulturellen Lernens, da eben in einem Land unterschiedliche Sprachen und kulturelle Werte- und Traditionsgemeinschaften vorhanden und damit zu berücksichtigen sind.

Vor dem elektronischen Zeitalter war es gar nicht immer so leicht, gewisse Informationen über fremde Sprach- und Kulturgemeinschaften zu erhalten. Heutzutage werden wir zwar mittels Internet und anderer Massenmedien von Reizen überflutet und eine der wichtigsten Aufgaben sowohl für private als auch für institutionelle Lernzwecke in Schule/Universität ist die entsprechende Auswahl der benötigten Information 
(bzw. von brauchbaren Texten und anderem Material) für die jeweilige Situation. Aber im Allgemeinen kommt die einfache Handhabung der Medien, die mit neuen Technologien verbunden sind, dem Lernen anhand von zielsprachigen Ressourcen entgegen. Myczko (2007: 177) sieht darin die Möglichkeit, selbstorganisiert und eigenverantwortlich in die fremde Wirklichkeit einzutauchen und mit der zielsprachigen Wirklichkeit und Kultur in Kontakt zu treten sowie kulturbezogene Phänomene zu verarbeiten.

Letztlich soll ja die Fähigkeit entwickelt werden,

die Ausgangskultur und die fremde Kultur miteinander in Beziehung zu setzen sowie die Fähigkeit, als kultureller Mittler zwischen der eigenen und fremden Kultur zu agieren und wirksam mit interkulturellen Missverständnissen und Konfliktsituationen umzugehen (M.U. Jańska 2006: 65).

\section{KULTUR - KULTURELLES LERNEN - INTERKULTURELLES POTENZIAL}

Wenn jemand etwas über eine fremde Kultur lernt, kann dies kulturelles Lernen genannt werden. Wenn zwei (oder mehr) Vertreter unterschiedlicher Kulturen von der jeweils anderen Kultur lernen, lernen sie inter-kulturell. Bei diesem Prozess der Interkulturalität lernen also beide Seiten voneinander. Kann man da die Tatsache, wie es beim Fremdsprachenunterricht meist der Fall ist, dass eine Seite, z.B. polnische Schülerinnen und Schüler, eine Fremdsprache lernen (und damit auch andere Elemente der fremden Kultur), schon als interkulturelles Lernen bezeichnen? Wird damit nicht fast schon jegliches Lernen interkulturell und damit das echte Interkulturelle Lernen inflationär?

$\mathrm{Ob}$ durch das Sprachenlernen automatisch auch ein interkulturelles Lernen, sprich das Kennenlernen, das Verstehen einer Fremdkultur stattfindet, das dann zu mehr Toleranz und Offenheit führen kann, ist mehr als fraglich. Oft genug führt ein sehr oberflächliches Verständnis ganz im Gegenteil zur Bestätigung, Verfestigung und Versteinerung von Stereotypen und (nationalen) Vorurteilen. Wohin das Kennenlernen einer anderen Kultur führt, hängt sicherlich von mehreren Faktoren ab, die in der Unterrichtssituation, bei den Lernenden aber auch bei den Lehrenden liegen können.

Wenn die Lehrenden kulturelle Elemente der Zielsprachenkultur vermitteln möchten, so müssen sie darauf vorbereitet sein - damit trägt eine entsprechende Lehrer/innen/ausbildung entscheidend dazu bei, ob eine durchschnittliche Lehrperson imstande sein wird, später als Sprachenleh- 
rer/in zur Entwicklung einer interkulturellen Kompetenz der Lerner/innen beizutragen oder nicht. Schon bei der Lehrer/innen/ausbildung muss es möglich sein, dass mutige Fragen gestellt werden, wobei didaktische Sicherheiten und Selbstverständlichkeiten manchmal in Frage gestellt oder sogar einstürzen können (vgl. Utri 2007: 128).

Alle diese auf kulturelle Tatsachen bezogene Unterrichtsmaterialien können potentielle interkulturelle Elemente ( $\mathrm{zu}$ Themen wie Rassismus, Diskriminierung von Minderheiten, Stereotype und Vorurteile, Migrationsphänomene, multikulturelles Miteinander usw.) sein, die zu einer positiven Entwicklung der interkulturellen Kompetenz einen Beitrag leisten können. Im Folgenden möchte ich zeigen, warum gerade Karikaturen, die meiner Ansicht nach oft ein hohes Motivations- als auch interkulturelles Potenzial aufweisen, für die Verwendung als didaktisches Material besonders geeignet sind. Somit erscheint es günstig und ratsam, diese in den interkulturellen Sprachenunterricht einzubauen.

\section{KENNZEICHEN VON KARIKATUREN}

Karikatur (von lateinisch carrus 'Karren', also: Überladung, und italienisch caricare 'überladen', 'übertreiben') bedeutet die komisch überzeichnete Darstellung von Menschen oder gesellschaftlichen Zuständen, auch mit politischem bzw. propagandistischem Hintergrund. Die Entsprechung im englischsprachigen Raum ist caricature ${ }^{1}$. Ähnliche Bezeichnungen sind Cartoon, Spottbild, Zerrbild, manchmal werden sie auch Scherzbild oder Scherzzeichnung genannt. Der Begriff Karikatur wurde von Philipp von Zesen durch den Ausdruck Zerrbild eingedeutscht.

Bei Karikaturen handelt es sich meist um eine bildliche Form der Satire, die sich als parteiische Kritik an bestehenden Werten oder politischen Verhältnissen versteht und oft als "Waffe” in gesellschaftlichen Auseinandersetzungen verwendet wird. Die Karikatur übertreibt bewusst, spitzt zu und verzerrt charakteristische Züge eines Ereignisses oder einer Person, um durch den aufgezeigten Kontrast zur Realität und die dargestellten Widersprüche den Betrachter der Karikatur zum Nachdenken zu bewegen. Oftmals nimmt die Karikatur zu einem aktuellen Sachverhalt sarkastischironisch Stellung. Wesentliche Fehler und Mängel der dargestellten Person (z.B. eines Politikers) oder des dargestellten Objektes oder Ereignisses werden aufgedeckt und durch die Art und Weise der meist zeichnerischen Präsentation der Lächerlichkeit preisgegeben.

\footnotetext{
${ }^{1}$ http:/ / de.wikipedia.org (10.09.2013)
} 
Die Karikatur kann mehr satirisch oder eher humoristisch ausgerichtet sein, je nachdem, ob sie ihr Opfer völlig verurteilt und lächerlich macht oder - als bloße Witzzeichnung - nur einige Mängel mit leiser Ironie kommentieren will. Bei einer Deutung von einer Karikatur geht man in folgenden Schritten vor: 1 . sich informieren, 2. beschreiben, 3. erklären und 4. zusammenfassen².

Karikaturen stecken nicht nur thematisch ein weites Feld ab. Sie beinhalten ein Bild, manchmal mit Text/Sprechblasen versehen und sind gezeichnet (somit ist auch die "Handschrift” des Künstlers sichtbar). Manchmal werden auch (gewisse) Bildgeschichten zu den Karikaturen gezählt (so berichtete Camilla Badstübner-Kizik auf dem IVG-Kongress im Juli 2010 in Warschau über das mangelhafte didaktische Beiwerk von Bildgeschichten; Genaueres zu Bild und Text in Badstübner-Kizik, 2006), manchmal Comics, jede größere Zeitung hat ihren Karikaturisten, der die (meist) tagespolitischen Ereignisse graphisch-satirisch aufs Korn nimmt.

\section{KARIKATUREN IN DER FREMDSPRACHENDIDAKTIK}

In der Fremdsprachendidaktik können Karikaturen auch sehr günstige Sprechanlässe bedeuten. Diese Methode eignet sich vor allem für zwei Dinge: „um durch Provokation zu motivieren” und um „Gedanken zu (aktuellen) Themen spontan einzubringen" (Schaefer 2011: 17).

Eine (deutsche) Karikatur, auf der z.B. eine Demonstration sichtbar ist, wo die Grünen auf die Straßen gehen, ist in Polen kaum denkbar, da die Grünen eine Partei sind, die im öffentlichen Leben (im Fernsehen und in anderen Medien) in Polen kaum präsent sind und von den meisten nur belächelt werden. Im Zuge des geplanten Baus von zwei Atomkraftwerken in Polen - wobei man hinzufügen muss, dass sich in den Gemeinden, die eventuell für einen Standort in Frage kämen, sehr wohl große Widerstände zeigen - könnte sich dies ändern und die belächelte "Umweltprotestpartei” könnte regen Zulauf bekommen.

Die Ironie des Schicksals will es, dass die Grünen im Rahmen der Koalition mit der Regierungspartei SPD über die allmähliche, aber endgültige Stilllegung aller deutschen Atomkraftwerke entschieden haben. Dieser Gegensatz (auch in der Mentalität) zwischen Deutschen und Polen kann im Unterricht genützt, zur Sprache gebracht und didaktisch "ausgeschlachtet" werden. Hier kommen nicht nur technische Problemstellungen zum Vorschein (Sicherheit, Entsorgung des Atommülls), sondern

\footnotetext{
2 http:/ / karikatur.augsten.at/definition-karikatur.php (10.09.2013).
} 
auch unterschiedliche Grundhaltungen zu dieser sog. friedlichen Nutzung der Kernenergie, deren Gefahr für Gesundheit und Leben in der deutschen und polnischen Gesellschaft jeweils in einer unterschiedlichen Intensität spürbar und sichtbar wird, wobei diese Haltungen auch an die junge Generation weitergegeben werden. Es ist anzunehmen, dass dies auch historisch bedingt ist. Die Deutschen, die sich seit Ende des Zweiten Weltkrieges technisch und marktwirtschaftlich recht schnell entwickelten, haben oft schon genug von der modernen Technik oder sehen zumindest schon die Risiken und Grenzen der Nutzung der Technik (daher auch solche Schlagworte wie Ethik in der Wirtschaft, soziale Technik usw.), die Polen hingegen, die ihre Wirtschaft (und damit auch Haushalte) erst ab den 1990-er Jahren modernisieren konnten, haben diese Phase noch (lange) nicht abgeschlossen (so heizen viele Haushalte auf dem Lande noch mit Holz oder Kohle) und sind noch immer sehr von Neuheiten/ neuen technischen Errungenschaften fasziniert. Natürlich ist diese Feststellung nicht verallgemeinerbar - sie sei eher als eine spürbare Tendenz gemeint - aber als provokativer Denkanstoß könnte sie im deutsch-polnischen Mentalitätsvergleich durchaus Verwendung finden.

\section{INTERKULTURELLE THEMEN IN KARIKATUREN}

Andere Themen, die in Polen im Fremdsprachenunterricht als didaktisches Mittel eher selten zur Sprache kommen, sind eben die interkulturellen Themen. Polen ist, abgesehen von einigen Regionen, in denen die Menschen andere Sprachen zusätzlich sprechen (Schlesien, die Kaschubei), sprachlich ein recht homogenes Land, es hat außerdem noch immer einen geringen Ausländeranteil in der Bevölkerung. Viele dieser Ausländer (v.a. aus der Ukraine, Weißrussland und Russland) haben sich darüber hinaus sprachlich und kulturell recht schnell eingegliedert, also werden in die polnisch-sprachige Gemeinschaft schnell aufgenommen bzw. können sich rasch assimilieren. Daher ist die Diskussion über Fremdländisches (in der BRD in der Folge die Diskussion z.B. über Leitkultur), über Ausländer in Polen (inklusive des Nutzens bzw. Schadens für das Land), deren (zum Teil schwierige/problematische) Integration und die vielleicht sinnvolle oder gar notwendige Förderung derselben in keinem sehr hohen Ausmaß vorhanden. Da die Tendenz, dass sich der Ausländeranteil erhöht, jedoch in fast allen europäischen Ländern gegeben ist, ist es sehr wohl sinnvoll, sich mit andersartigen Kulturen (oder auch Religionen/ Konfessionen) zu befassen. Es scheint daher günstig zu sein, wenn sich die heutige polnische Jugend schon gedanklich damit befasst und 
sich damit auf eine solche Situation zumindest geistig schon vorbereitet. Ein Anlass dafür kann der fremdsprachliche Unterricht sein.

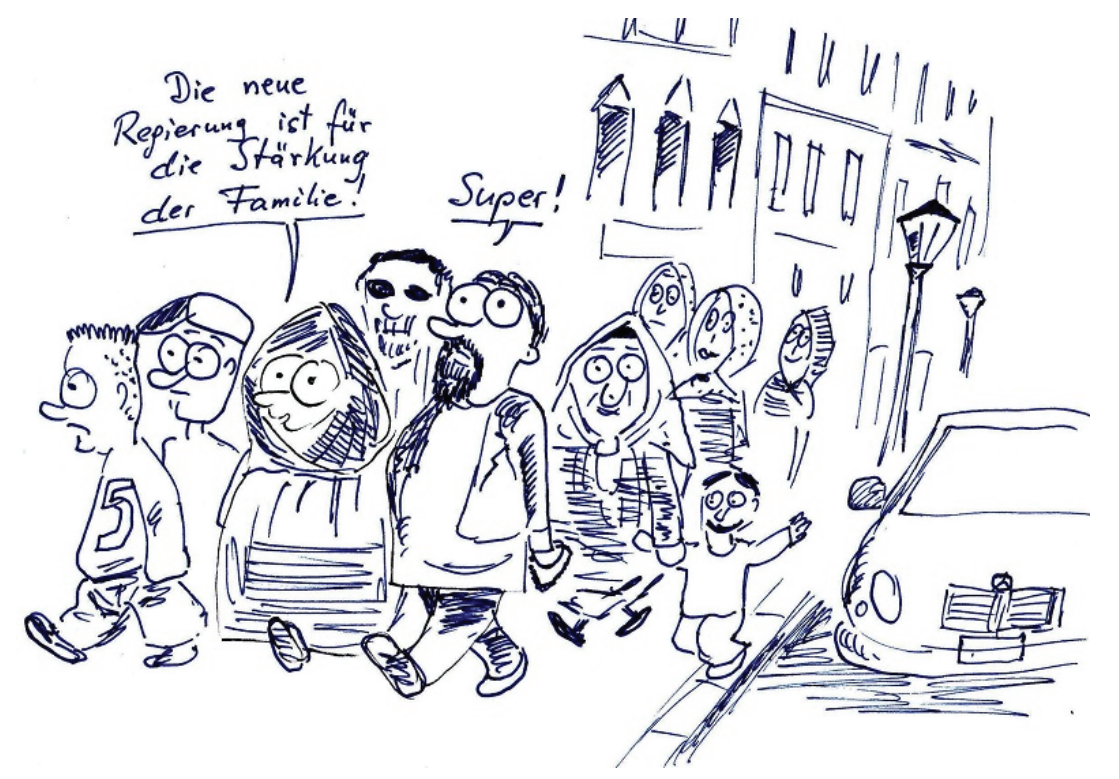

Zeichnung: der Verfasser

Durch so eine Karikatur können Fragen nach sprachlichen/religiösen Minderheiten aufgegriffen werden oder auch die Frage nach der effektiven Eingliederung von vielen Immigranten in die Gesellschaft (Gefahren und Chancen, Veränderungen) diskutiert werden. Sie eignen sich auch vorzüglich als Einleitung bzw. Auflockerung bei Übersetzungsübungen, bei denen Texte mit ähnlichem Inhalt zum Tragen kommen. An Karikaturtexten können auch viele andere sprachliche Phänomene besprochen und gelernt werden (z.B. die Polysemie von Wörtern = zwei oder mehrere Bedeutungen). Durch das bildlich-humorvolle Anschauungsmaterial ist die Chance größer, dass die jeweiligen Bedeutungen/ Erklärungen bzw. der Sprachwitz in dem Text besser eingeprägt werden.

Hier zwei Beispiele: Es wird eine Raumstation skizziert, wo ein Astronaut zum anderen sagt: „Hier, ziehen Sie sich die Magnetschuhe an, damit Sie wieder einen festen Standpunkt haben". Und auf dem nächsten Bild, als der Kollege mit den Magnetschuhen an der Wand schon ein Stück hochgegangen ist, meint dieser: "Mit diesen Dingern kann sogar der friedlichste Mensch die Wände hochgehen."

Oft werden Sprachspiele in Karikaturtexten eingebaut, um die Kariakturen - z.B. in Comics - interessanter, sprich intelligent und witzig 
zu gestalten. Z.B. tritt dies häufig in Asterix und Obelix auf; im Folgenden ein kleines Beispiel3:

„Da haben wir den Salat, Asterix. Und das alles wegen dem bisschen Öl!" "Genau! Jetzt aber Beeilung, wir müssen einen Heilpraktiker finden. Sonst ist alles Essig!".

Karikaturen sind - das soll hier klar ersichtlich werden - vielfältig einsetzbar, und nicht nur als Auflockerungseinheit bzw. Einstiegsübung. Im Fach, das sich „Kreatives Schreiben” nennt, können Karikaturen in Form ganzer Cartoons präsentiert und später von den Lernenden selbst entwikkelt werden. In einer Gruppe von StudentInnen finden sich immer ein paar, die Übung im Zeichnen haben. Zu diesen Zeichnungen können dann z.B. lustige Verse dazugedichtet werden.

Die ernstesten Themen können damit behandelt werden. Im Folgenden sehen wir zwei Karikaturen, ${ }^{4}$ welche die Diskussion über den Islamunterricht in den deutschen/ österreichischen Schulen aufzeigt sowie die weitverbreitete Meinung vieler von den Medien einseitig informierter Menschen über die Allgemeingefährlichkeit aller Islamisten betrifft. Es gibt diesbezüglich viele vorschnell gefasste Urteile (also Vorurteile) und negative Vorstellungen - alle Anhänger des Islams, egal, wie moderat oder extremistisch sie seien, werden da in einen Topf geworfen.
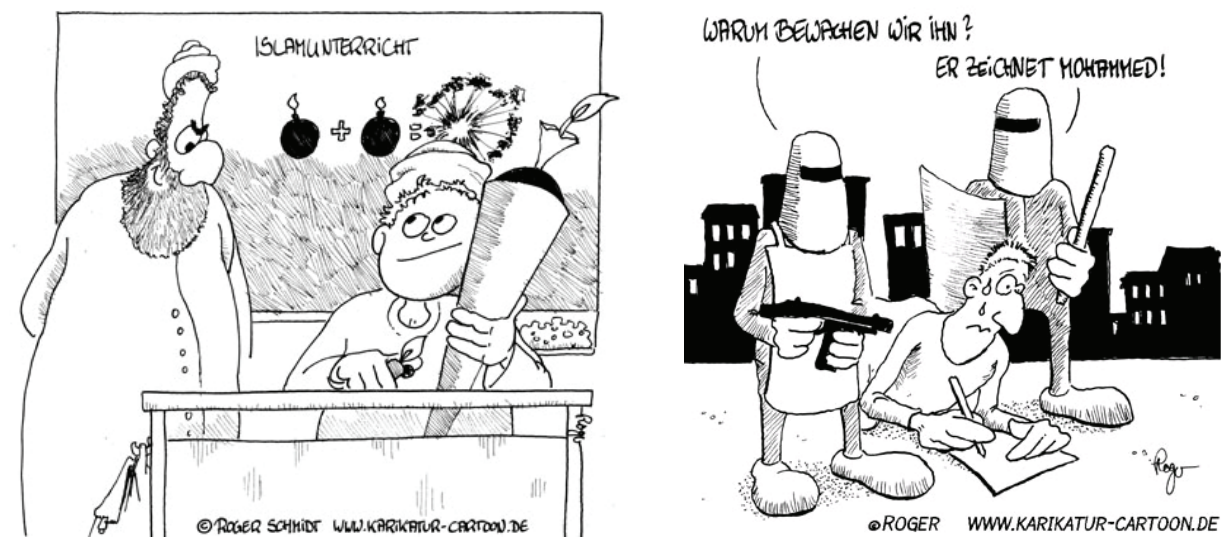

\footnotetext{
${ }^{3}$ vgl. http://www.comedix.de/lexikon/special/sprachspiel/die_odyssee.php (10.09.2013).

${ }^{4}$ Quellen: http://www.brainworker.ch/Irak/Islam.htm, bzw. http://www.karikaturcartoon.de/politik/islamunterricht.htm (10.09.2013).
} 
Solche Karikaturen können $\mathrm{zu}$ unterschiedlichen Themen erarbeitet werden, in Form von schriftlichen Aussagen dazu, in Form von Diskussionsbeiträgen oder auch in Form von Bildbeschreibungen. Hier können unterschiedliche (hier z.B. religiöse Vorstellungen) fremdkulturellen Bezüge herausgearbeitet werden, und die allgemeine kulturell-zivilisatorische Entwicklung der islamischen Welt (inklusive der potenziellen Gefahr von Attentätern) kann angesprochen und reflektiert werden. Gerade bei solchen heiklen Themen soll man jedoch bedacht sein, nicht einseitig zu werden diese Einseitigkeit sieht man ja oft genug in den Boulevard-Blättern der Tagespresse. Hier würden zum Beispiel schriftliche Übungen günstig sein, die daraufhin abzielen, dass sich die Lernenden in die Lage eines Fremden (hier: Anhänger des Islam) hineinzuversetzen haben.

Man kann nur hoffen, dass sich immer mehr Lehrerinnen und Lehrer die (eigentlich geringe) Mühe machen, hin und wieder ein paar Karikaturen in den Unterricht mitzubringen und sich mit diesen zusammen mit den SchülerInnen bzw. StudentInnen auseinanderzusetzen.

\section{LITERATURVERZEICHNIS}

Badstübner-Kizik, C., 2006. Fremde Sprachen - fremde Künste? Bild- und Musikkunst im interkulturellen Fremdsprachenunterricht. Das Fallbeispiel Deutsch als Fremdsprache in Polen. Gdańsk: Wyd. UG.

Grucza, F., 1993. Zum Begriff der interkulturellen Verständigungskompetenz und über die Notwendigkeit ihrer Pflege. In: Gamkrelidze, Th.V. et al. (Hrsg.) Brükken. Festgabe für Gert Hummel zum 60. Geburtstag. Tbilisi-Konstanz, Verlag der Djawachis-chwili Staats-uniwersität/Konstanz: Universitäts-verlag. 147-172.

Grucza, S., 2006. Idiolekt specjalistyczny - Idiokultura specjalistyczna - Interkulturowość specjalistyczna. W: Lewandowski, J., Kornacka, M., Woźniakowski, W: Języki Specjalistyczne 6. Teksty specjalistyczne w kontekstach międzykulturowych i tłumaczeniach, Katedra Języków Specjalistycznych. Warszawa: Uniwersytet Warszawski, 30-49.

Jańska, M.U., 2006. Interkulturelles Lernen In der bilingualen deutsch-polnischen Erziehung. Evaluation der Unterrichtsmaterialien für den frühen fremdsprachlichen Deutschunterricht in Polen. Reihe Dissertationes Inaugurales Selectae. WrocławDresden: Oficyna Wydawnicza ATUT, Neisse Verlag.

Krumm, H.-J., 2003. Einführung: Der Beitrag der Universitäten zur europäischen Sprachenvielfalt. In: Krumm H.-J. (Hrsg.) Sprachenvielfalt. Babylonische Sprachverwirrung oder Mehrsprachigkeit als Chance? Innsbruck-Wien-München-Bozen, Studien Verlag.

Myczko, K., 2007. Intermedialität im Fremdsprachenlernen und -lehren. In: Grucza, F., Schwenk, H.-J., Olpińska, M. (Hrsg.), Germanistische Wahrnehmungen der Multimedialität, Multilingualität und Multikulturalität. Materialien der Jahresta- 
gung des Verbandes Polnischer Germanisten 11.-13. Mai 2007. Opole- Warszawa: Wyd. Euro-Edukacja, 175-182.

Pfeiffer, W., 2001. Von der linguistisch bezogenen Fremdsprachendidaktik zur interkulturellen Fremdsprachenpädagogik. Essay. http://www.linguistik-online.de/13_01/ pfeiffer.html.

Röttger, E., 2010. Interkulturelles Lehren und Lernen in der Unterrichtspraxis DaF: Grenzüberschreitung oder Hürdenlauf? In: Zeitschrift für Interkulturellen Fremdsprachenunterricht, Jahrg. 15: 2, 7-24. Abrufbar unter http://zif.spz.tudarmstadt.de/jg-15-2/beitrag/Roettger.pdf.

Schaefer, St., 2011. Unterrichtsmethoden. Schwerpunkt kaufmännische Fächer, HaanGruiten Verlag Europa-Lehrmittel, Nourney, Vollmer GmbH \& Co.

Utri, R., 2007. Interkulturalität und Fremdsprachenlernen in Österreich, Deutschland und Polen vor dem Hintergrund der Sprachenpolitik der Europäischen Union. Unveröff. Dissertation, Universität Warschau.

Utri, R., 2008. Filmy jako możliwość interkulturowego uczenia się - na podstawie amerykańskiego filmu "Moje wielkie greckie wesele" i austriackiego filmu „I love Vienna". In: Przeglad Glottodydaktyczny 25, 107-116.

Utri, R., 2009a. Poesie als schöpferisches Element in der Glottodidaktik/Litera turkunde. In: Studia Niemcoznawcze XL, 417-436.

Utri, R., 2009b. Sprache geht ins Ohr, über Musik even more - zur Verwendung von musikalischen Mitteln in der interkulturellen Glottodidaktik. In: Studia Niemcoznawcze XLII, 323-335.

Utri, R., 2010a. Das kreativ-interkulturelle Potenzial von Drama-Techniken in der Glottodidaktik. In: Studia Niemcoznawcze XLV, 515-524.

Utri, R., 2010b. Deutsch-türkische Freundschaft: über die Notwendigkeit des Einbindens der Migrantenliteratur in das interkulturelle Lernen. In: Glottodidactica. An International Journal of Applied Linguistics XXXVI, 211-223.

Utri, R., 2010c. Migrantenliteratur im DaF-Unterricht: Potenzial für das interkulturelle Lernen. In: Lingwistyka Stosowana / Applied Linguistics / Angewandte Linguistic 3, 279-290.

\section{Internetadressen}

http:/ / karikatur.augsten.at/definition-karikatur.php

http://www.comedix.de/lexikon/special/sprachspiel/die_odyssee.php

http://www.brainworker.ch/Irak/Islam.htm

http://www.karikatur-cartoon.de/politik/islamunterricht.htm 
